\title{
Continuous reversal using internal or external cues: A novel test measuring set shifting in Parkinsonian rats
}

\author{
Thibaut Sesia $^{\text {a,b,d, }}$, Rob Hameleers ${ }^{\mathrm{a}, \mathrm{b}, \mathrm{c}}$, Rinske Vlamings ${ }^{\mathrm{a}, \mathrm{b}, \mathrm{d}}$, Eva Wölbert ${ }^{\mathrm{c}}$, Süleyman Kaplan ${ }^{\mathrm{e}}$, Arjan Blokland ${ }^{\mathrm{a}, \mathrm{b}, \mathrm{d}}$ and Yasin \\ Temel*a,b,d
}

\author{
${ }^{a}$ Department of Neuroscience, Faculty of Health, Medicine and Life Sciences, Maastricht University, The Netherlands \\ ${ }^{b}$ Department of Neurosurgery, Maastricht University Medical Center, The Netherlands \\ 'Department of Neuropsychology and Psychopharmacology, Faculty of Psychology and Neuroscience, Maastricht University, \\ The Netherlands \\ 'European Graduate School of Neuroscience (EURON) \\ ${ }^{e}$ Department of Histology and Embryology, Medical School, Ondokuz Mayls University, Samsun, Turkey
}

\begin{tabular}{|c|c|c|}
\hline \multicolumn{2}{|c|}{ ARTICLE INFO } & ABSTRACT \\
\hline \multicolumn{2}{|c|}{ Article History } & \multirow{11}{*}{$\begin{array}{l}\text { Parkinson's disease (PD) is a predominant movement disorder, but profound cognitive } \\
\text { deficits (e.g. bradyphrenia, memory, and set shifting) also occur. To model the deficits in } \\
\text { set shifting using internal and external cues in rats we developed a continuous reversal } \\
\text { task in which the active lever, left or right lever, alternated after a variable number of } \\
\text { lever presses. In one task the active lever was signaled by a light (external cue condition, } \\
\text { EC) whereas in the other task the active lever was not signaled (internal cue condition, } \\
\text { IC). In this study we evaluated the effects of a partial bilateral striatal 6-OHDA lesion as } \\
\text { model for PD on the performance in both tasks. Following behavioral testing the lesions } \\
\text { were verified using tyrosine hydroxylase (TH) immunohistochemistry. The 6-OHDA } \\
\text { lesioned animals were specifically impaired in the IC condition and not in the EC task. In } \\
\text { other words, the lesioned animals kept pressing a lever longer although it was not longer } \\
\text { active. The present response switching task is sensitive to 6-OHDA lesions and may } \\
\text { mimic set-shifting deficits in PD. } \\
\text { J. Exp. Clin. Med., 2012; 29:183-186 }\end{array}$} \\
\hline ved & $12 /$ & \\
\hline Accepted & & \\
\hline \multirow{7}{*}{\multicolumn{2}{|c|}{$\begin{array}{l}\text { * Correspondence to: } \\
\text { Yasin Temel } \\
\text { Department of Neurosurgery, } \\
\text { Maastricht University Medical Center } \\
\text { P. Debyelaan 25, 6202 AZ Maastricht, } \\
\text { The Netherlands } \\
\text { e-mail: y.temel@maastrichtuniversity.nl }\end{array}$}} & \\
\hline & & \\
\hline & & \\
\hline & & \\
\hline & & \\
\hline & & \\
\hline & & \\
\hline & & \\
\hline
\end{tabular}

Set-shifting

Continuous reversal

6-OHDA lesion

Parkinson's disease

(C) 2012 OMU

\section{Introduction}

Parkinson's disease (PD) is a progressive neurodegenerative disorder that is generally determined by motor disturbances such as slowness of movement, difficulty in initiating movement, rigidity and tremor (Fahn, 2003). However, PD is also associated with cognitive deficits (Owen, 2004). Amongst different cognitive dysfunctions, PD patients particularly have problems with cognitive set-shifting tasks, i.e. the ability to flexibly adapt behavior in response to environmental changes. Hayes et al. (1998) found significantly increased reaction times due to set shifting problems in PD patients. Moreover, it has been suggested that PD patients have a greater difficulty when response switches are based on internal cues as opposed to external cues (Brown and Marsden, 1988; Hayes et al., 1998).

To further investigate the neurobiological mechanisms underlying these cognitive impairments in PD and to evaluate possible treatments, an animal model that mimics the set shifting deficits in PD may offer great advantages.
Cognitive set-shifting in rodents has been assessed by various experimental setups in the past. A cross maze was used to examine shifting between place and response learning (Ragozzino et al., 1999) or shifting between response and cue learning (Floresco et al., 2006). Birrel and Brown (2000) applied a design in which rats had to shift between discrimination by odor, digging medium and texture allowing for reversal learning, intradimensional and extradimensional shifting to be discriminated. Most of these studies focus on the role of the prefrontal cortex in set-shifting. Prefrontal cortex activation however is mediated by the basal ganglia via the so-called associative circuit (Alexander et al., 1990), suggesting vulnerability of frontal functions at this level (Alexander et al., 1990).

By inactivation of the dorsomedial striatum, Ragozzini, Jih and Tzavos (2002) showed that this brain region is involved in set-shifting. Injection of the local anesthetic bupivacaine did not affect initial acquisition, but reversal learning. More specifically the deficit was not found in abandoning the 
previously learned strategy, but in maintaining the new strategy.

To further increase practicability of this approach the aim of the present study was to develop a sensitive Skinner box test for cognitive set-shifting in rats.

\section{Material and methods \\ Animals}

All animal surgical and maintenance procedures were approved by the local ethics committee of Maastricht University and met government guidelines. Sixteen 4-month-old male Wistar rats were housed in standard Macrolon (type III) cages bedded with sawdust. The room was air-conditioned to about $21^{\circ} \mathrm{C}$. During behavioral testing in the skinner boxes, in which the animals were reinforced with food pellets, rats received restricted feeding with $12 \mathrm{~g}$ of laboratory chow per day and ad libitum access to water in their cages at all times. In the weekend, rats had ad libitum access to food until Sunday evening, so that their weight was about $90 \%$ of their ad libitum weight. Testing took place in the dark-phase of a reversed light-dark cycle. Ten animals received 6-OHDA lesion, and six sham-operated animals served as controls. Three 6-OHDA animals died during or shortly after surgery.

\begin{abstract}
Apparatus
Animals were trained in 9 identical operant chambers (inner dimensions: 40x30x33 cm) that were equipped with two retractable levers, and cue lights just above the levers. Levers were $4 \mathrm{~cm}$ wide and protruded $2 \mathrm{~cm}$ into the conditioning. chamber. Their position was $12 \mathrm{~cm}$ above the grid floor with $12 \mathrm{~cm}$ in between them. Reward was delivered into a food tray ( $5 \times 5 \mathrm{~cm}$ and $2.5 \mathrm{~cm}$ above the grid floor), that was placed centered between the two levers, and could be accessed by pushing a hinged panel.
\end{abstract}

\section{Behavioral procedure}

Rats were trained on two versions of a response switching task. In both tasks, two levers were presented, but only one was reinforced. Further, a fixed ratio 5 (FR5) schedule was applied on all trials. Switching of the reinforced lever occurred after a variable number of reinforcements. On average a switch in the reinforced lever occurred after five reinforcements (i.e. 25 lever presses on one lever). A total of 80 reinforcements could be achieved (equally divided over left and right lever). Pressing of the inactive lever in a series of lever presses on the active lever resulted in restarting of the FR 5 schedule on the active lever.

Training took place twice a day in session of about 20 min. Two stimulus conditions were introduced: Internal cue condition (IC), where no cues were presented to indicate the switch in the reinforced lever, and external cue condition (EC), where a light above the lever served as a cue reinforced to indicate the active lever (Fig. 1). All rats were first trained on the IC task until no further change in performance (see below for behavioral measures) was observed, and subsequently on the EC task, again until performance was stable, i.e. no change in performance on six consecutive sessions. Then, a final pre-surgery testing series on the IC task took place. Following the surgery the same series of pre-surgery testing was repeated (Fig. 1).

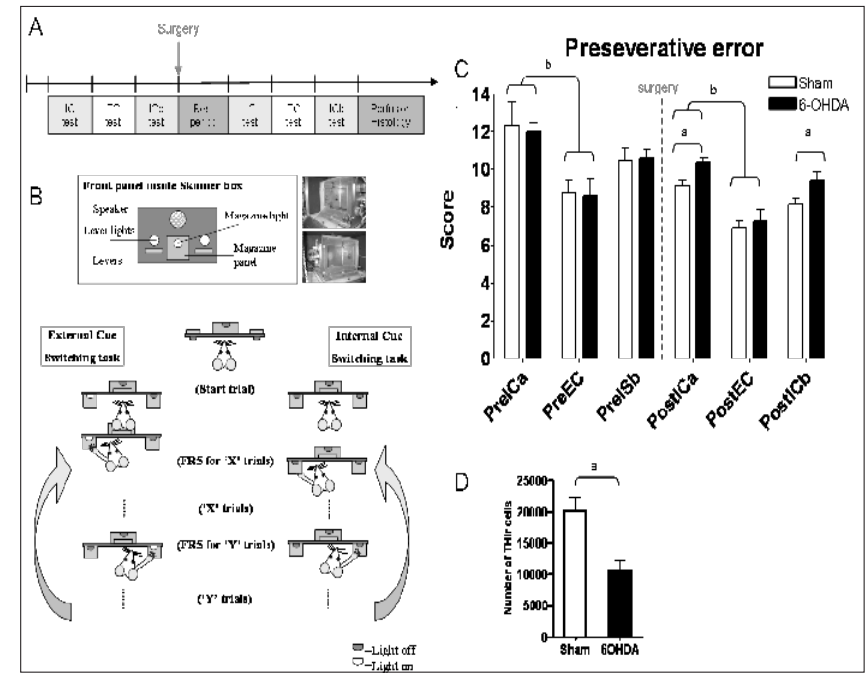

Fig. 1. The overview of the experiment, (A) presents the schedule of the entire experiment, and (B) shows a scheme that summarizes the IC/EC set-shifting task. The results of the behavior and the histology for both groups are respectively in (C) and (D), as mean \pm SEM of the PEs and number of THir counted cell. preIC: IC test presurgery, preEC: EC test presurgery, PreICb: second IC test presurgery, postIC: IC test postsurgery, postEC: EC test postsurgery, PostICb: second IC test postsurgery; a: statistical differences $(p<0.05)$ between sham and lesioned group, b: statistical differences between IC and EC test.

Behavioral performance was assessed using three parameters:

- Perseveration errors (PEs): Total number of lever presses on the previous reinforced lever after the active lever was switch to the other side.

- Regressive errors (REs): Total number of lever presses to the previously reinforced lever after the newly reinforced lever had been pressed at least once.

- Time to reward (TR): Time that was needed to obtain the first reward after a switching event occurred.

\section{Surgical procedure}

Rats were divided into two groups of matched body weight and prior performance measured in amount of perseveration errors. During the whole surgical procedure the animals were anesthetized with a combination solution of ketamine (90 $\mathrm{mg} / \mathrm{kg} \mathrm{s.c)}$ and xylazine (10 mg/kg s.c) before the surgery. The experimental group received desmethylimipramine (20 $\mathrm{mg} / \mathrm{kg}$ in $0.9 \%$ saline, volume $2 \mathrm{ml} / \mathrm{kg}$, s.c) one hour before anesthesia in order to block serotonin and norepinephrine uptake and make the lesion more specific for the dopaminergic system. Rats were injected bilaterally at two sites per hemisphere with a solution of $6 \mu \mathrm{g} / \mu \mathrm{l} 6-\mathrm{OHDA}$ in $0.9 \% \mathrm{NaCl}$ and $0.2 \%$ ascorbic acid. The volume of each injection was $2 \mu 1$ and was delivered using a micropump with a speed of $1 \mu \mathrm{l} /$ min. After the injection, the canulla was left in place for $2 \mathrm{~min}$ in order to allow the solution to diffuse into the tissue. Injection sites were +0.7 anterior to bregma, $\mu 2.8$ lateral to midline, -5.0 ventral from the skull, and -0.4 posterior to bregma, $\mu 3.4$ lateral, -5.0 ventral from the skull, respectively. The control group received sham operation, i.e. rats were treated similar to the experimental group, but were injected with saline instead of 6-OHDA. 


\section{Histological processing}

At the end of the testing, rats were brought to deep anaesthesia with an overdose of pentobarbital and then perfused transcardially with tyrode $(0.1 \mathrm{M})$ and fixative containing $4 \%$ paraformaldehyde, $15 \%$ picric acid and $0.05 \%$ glutaraldehyde in $0.1 \mathrm{M}$ phosphate buffer ( $\mathrm{pH}$ 7.6). Brains were removed and postfixed for $2 \mathrm{~h}$ followed by overnight immersion in $15 \%$ sucrose at $4^{\circ} \mathrm{C}$. Brain tissue was then quickly frozen with $\mathrm{CO}_{2}$ and stored at $-80^{\circ} \mathrm{C}$. Subsequently, the brains were cut serially on a cryostat into $30 \mu \mathrm{m}$ frontal sections and again stored at $-80^{\circ} \mathrm{C}$. Tyrosine hydroxylase $(\mathrm{TH})$ immunohistochemistry was carried out using mouse anti-TH (diluted 1:2000, kindly supplied by Dr. C. Cuello, Canada) as primary antibody (Temel et al., 2006). After rinsing steps with Tris-buffered solution (TBS), TBS, TBS-Triton X-100 and incubation with the secondary antibody (diluted 1:400 donkey anti-mouse biotin; Jackson Immunoresearch Laboratories, West Grove, USA), the sections were incubated with ABC-kit (diluted 1:800, Vector laboratories, USA). To visualize the horseradish peroxide (HRP) reaction product, the sections were incubated with 3,3'-diaminobenzidine tetrahydrochloride (DAB).

\section{Stereological analysis}

Stereological analysis was performed with a stereology workstation (CAST-GRID-Computer Assisted Stereological Toolbox, Olympus, Denmark). After exactly tracing the boundaries of the substantia nigra pars compacta ( $\mathrm{SNc}$ ) on microscopic video images displayed on a monitor, numbers of tyrosine hydroxylase immunoreactive (THir) neurons and total numbers of neurons were evaluated with the optical fractionator technique. All neurons whose the largest border of nucleus came into focus within unbiased virtual counting spaces distributed in a systematic-random fashion throughout the delineated regions were counted. Estimated numbers of neurons were calculated from the numbers of counted neurons and the corresponding sampling probability (Temel et al., 2006; Ayyildiz et al., 2008).

\section{Statistical analysis}

Behavioral measures were averaged across animals for each training session. Cuing and lesion effects on behavior and THir cells in the SNc were tested via an independent-samples Student t-test. Data were analyzed using SPSS statistical software (version 15.0). A p-value lower than 0.05 was considered to be statistically significant.

\begin{tabular}{|c|c|c|c|c|}
\hline & \multicolumn{2}{|c|}{ Regression Errors } & \multicolumn{2}{|c|}{ Time to Reward (s) } \\
\hline & 6-OHDA & Sham & 6-OHDA & Sham \\
\hline Pre ICa & $1.19 \pm 0.358$ & $1.03 \pm 0.490$ & $1.22 \pm 0.17$ & $1.18 \pm 0.17$ \\
\hline Pre EC & $0.08 \pm 0.025$ & $0.07 \pm 0.016$ & $0.86 \pm 0.05$ & $0.82 \pm 0.10$ \\
\hline Pre Icb & $0.19 \pm 0.081$ & $0.09 \pm 0.031$ & $1.29 \pm 0.32$ & $0.77 \pm 0.06$ \\
\hline Post ICa & $0.18 \pm 0.063$ & $0.28 \pm 0.101$ & $1.24 \pm 0.20$ & $1.03 \pm 0.20$ \\
\hline Post EC & $0.04 \pm 0.011$ & $0.06 \pm 0.008$ & $0.67 \pm 0.08$ & $0.80 \pm 0.18$ \\
\hline Post Icb & $0.11 \pm 0.025$ & $0.09 \pm 0.019$ & $1.22 \pm 0.31$ & $0.66 \pm 0.06$ \\
\hline
\end{tabular}

\section{Results}

The results of the behavior and the histology are presented table 1 and illustrated respectively figure 1C and 1D (Table $1)$.

\section{Cuing}

To compare the effect of the cuing we compared EC and IC separately in both groups pre- and post surgery. The tests revealed that in both groups and before and after surgery animals made significantly less PEs in the EC as compared IC version of the test. The sham rats made $29 \%$ less PEs before surgery (IC vs. EC: $\mathrm{t}(6)=3.50, \mathrm{p}<0.02$ ) and $23 \%$ less post surgery $(\mathrm{t}(4)=5.80, \mathrm{p}<0.01)$. The 6-OHDA lesioned group made $31 \%$ less PEs before surgery $(\mathrm{t}(6)=4.12, \mathrm{p}<0.01)$, and $30 \%$ post surgery $(\mathrm{t}(6)=4.03, \mathrm{p}<0.01)$.

\section{Lesion effect}

No lesion effects were found on the parameters time to reward (t's $<1.894$, n.s.) and regression errors (t's $<1.160$, n.s.) in the IC and EC test conditions post surgery. However, the lesioned animals made more PEs than the sham animals in the post surgery IC tests $(t(11)=2.21, p<0.05)$, but not in the post $\mathrm{EC}$ test condition $(\mathrm{t}(11)=0.42$, ns.).

The lesioned rats performed as well as sham with regards to the EC test. However, the parkinsonian rats performed worse on the IC test, which is in line with previous report linking dopamine depletion and impaired performance in internally based cued action (Moustafa et al., 2008; NaganoSaito et al., 2008). This finding is in line with our hypothesis that the present paradigm assesses impairment in flexibility in Parkinsonian patient (Brown and Marsden, 1988; Brown and Marsden, 1991; Owen et al., 1993; Cools et al., 2001; Witt et al., 2006; Polito et al., 2007).

\section{Dopamine lesion}

The efficacy of the lesion was estimated by the amount of cell loss within the SNc. A significant loss (47\%) of THir positive cells was found in the lesioned group $(\mathrm{t}(11)=3.50, \mathrm{p}<0.01)$. This level of cell loss mimics pre-symptomic phase of the disease, while symptoms start to appear between $60-80 \%$ of dopamine loss in animal model. $80 \%$ of loss is usually considered as the onset of the symptom in human patient (Kirik et al., 1998; Ling et al., 2000). However, it should be noted that in rats the time course of 6-OHDA induced dopamine deafferentiation is different from that of PD (Deumens et al., 2002). Thus, it may be suggested that the effects observed in the present study underestimate the effects of greater DA depletions. On the other hand, these findings suggest that this test is sensitive enough to pick up behavioral effects at a DA loss of about $50 \%$.

\section{Discussion}

The IC/EC set-shifting test used in the present study showed that rats made less PEs in the EC condition as compared to the IC condition. Thus, rats made about 3-4 more PEs in the IC condition as compared to the EC condition. Further, our study shows that this task was able to pick up a specific effect of moderate (50\%) DA lesions on IC switching. Thus, no effects were observed on EC and other parameters in this task. These effects in parkinsonian rats are assumed reflecting the deficits in set shifting as observed in PD patient. On basis 
of the present study we conclude that the present paradigm could be used as a relative easy test to examine set shifting in animal models with fronto-striatal lesions, such as in PD.

\section{REFERENCES}

Alexander, G.E., Crutcher, M.D., DeLong, M.R., 1990. Basal ganglia-thalamocortical circuits: Parallel substrates for motor, oculomotor, "prefrontal" and "limbic" functions. Prog. Brain Res. 85, 119-146.

Ayyildiz, M., Kozan, R., Agar, E., Kaplan, S., 2008. Sexual dimorphism in the medial vestibular nucleus of adult rats: Stereological study. Anat. Sci. Int. 83, 131-139.

Birrell, J.M., Brown, V.J., 2000. Medial frontal cortex mediates perceptual attentional set shifting in the rat. J. Neurosci. 20, 4320-4324.

Brown, R.G., Marsden, C.D., 1988. Internal versus external cues and the control of attention in Parkinson's disease. Brain. 111, $323-345$.

Brown, R.G., Marsden, C.D., 1991. Dual task performance and processing ressources in normal subjects and patients with Parkinson disease. Brain. 114, 215-231.

Cools, R., Barker, R.A., Sahakian, B.J., Robbins, T.W., 2001. Mechanisms of cognitive set flexibility in Parkinson's disease. Brain. 124, 25032512.

Deumens, R., Blokland, A., Prickaerts, J., 2002. Modeling Parkinson's disease in rats: An evaluation of 6-OHDA lesions of the nigrostriatal pathway. Exp. Neurol. 175, 303-317.

Fahn, S., 2003. Description of Parkinson's disease as a clinical syndrome. Ann. NY Acad. Sci. 991, 1-14.

Floresco, S.B., Ghods-Sharifi, S., Vexelman, C., Magyar, O., 2006. Dissociable roles for the nucleus accumbens core and shell in regulating set shifting. J. Neurosci. 26, 2449-2457.

Hayes, A.E., Davidson, M.C., Keele, S.W., Rafal, R.D., 1998. Toward a functional analysis of the basal ganglia. J. Cogn. Neurosci. 10, 178198.

Kirik, D., Rosenblad, C., Björklund, A., 1998. Characterization of behavioral and neurodegenerative changes following partial lesions of the nigrostriatal dopamine system induced by intrastriatal 6-hydroxydopamine in the rat. Exp. Neurol. 152, 259-277.

Ling, Z.D., Collier, T.J., Sortwell, C.E., Lipton, J.W., Vu, T.Q., Robie, H.C., Carvey, P.M., 2000. Striatal trophic activity is reduced in the aged rat brain. Brain Res. 856, 301-309.

Moustafa, A.A., Sherman, S.J., Frank, M.J., 2008. A dopaminergic basis for working memory, learning and attentional shifting in Parkinsonism. Neuropsychologia. 46, 3144-3156.

Nagano-Saito, A., Leyton, M., Monchi, O., Goldberg, Y.K., He, Y., Dagher, A., 2008. Dopamine depletion impairs frontostriatal functional connectivity during a set-shifting task. J. Neurosci. 28, 3697-3706.

Owen, A.M., 2004. Cognitive dysfunction in Parkinson's disease: The role of frontostriatal circuitry. Neuroscientist. 10, 525-537.

Owen, A.M., Roberts, A.C., Hodges, J.R., Summers, B.A., Polkey, C.E., Robbins, T.W., 1993. Contrasting mechanisms of impaired attentional set-shifting in patients with frontal lobe damage or Parkinson's disease. Brain. 116, 1159-1175.

Polito, C., Ramat, S., 2007. 3.116 Set-shifting deficit and fronto-temporal hypometabolism in early Parkinson's disease. Parkinsonism Relat. D. $13,157-157$

Ragozzino, M.E., Detrick, S., Kesner, R.P., 1999. Involvement of the prelimbic-infralimbic areas of the rodent prefrontal cortex in behavioral flexibility for place and response learning. J. Neurosci. 19, 4585-4594.

Ragozzino, M.E., Jih, J., Tzavos, A., 2002. Involvement of the dorsomedial striatum in behavioral flexibility: Role of muscarinic cholinergic receptors. Brain Res. 953, 205-214.

Temel, Y., Visser-Vandewalle, V., Kaplan, S., Kozan, R., Daemen, M.A., Blokland, A., Schmitz, C., Steinbusch, H.W., 2006. Protection of nigral cell death by bilateral subthalamic nucleus stimulation. Brain Res. 1120, 100-105.

Witt, K., Daniels, C., Schmitt-Eliassen, J., Kernbichler, J., Rehm, S., Volkmann, J., Deuschl, G., 2006. The impact of normal aging and Parkinson's disease on response preparation in task-switching behavior. Brain Res. 1114, 173-182. 